\title{
Fibroblasts mediate induction of high mobility group box protein 1 in lung epithelial cancer cells by diffusible factors
}

\author{
BABETT BARTLING, CHRISTINE FUCHS, ROLF-EDGAR SILBER and ANDREAS SIMM
}

\author{
Department of Cardiothoracic Surgery, Martin Luther University Halle-Wittenberg, D-06120 Halle/Saale, Germany
}

Received March 27, 2007; Accepted May 2, 2007

\begin{abstract}
Cancer development is associated with the high mobility group box protein 1 (HMGB1), which modulates the transcriptional activity in the nucleus, but it is also present in the cytoplasm and outside the cell in certain conditions. As the progression of lung cancer is supported by mitogenic stimuli of stromal fibroblasts, we studied the impact of lung fibroblasts (WI-38) on the expression and localization of HMGB1 in lung epithelial cancer cells (H358). HMGB1 was mainly localized in the nucleus of non-mitotic H358 cells but highly distributed in the cytoplasm of mitotic cells. Conditioned medium (CM) from WI-38 fibroblasts enhanced the expression of HMGB1 at the mRNA and protein level compared to the control CM from H358 cells. In particular, the amount of cytoplasmic HMGB1 was elevated in response to fibroblast $\mathrm{CM}$, which was reduced by inhibiting the basic fibroblast growth factor with blocking antibodies. Although cytoplasmic HMGB1 can be released from the cell, we did not determine a significant amount of extracellular HMGB1 in these conditions. This might partially be based on the sensitivity of HMGB1 to extracellular proteases as $\mathrm{CM}$ caused fast proteolysis of the cytoplasmic HMGB1 preparations. Our data suggest that diffusible factors of fibroblasts support the biological function of cancer cells via HMGB1 activation.
\end{abstract}

\section{Introduction}

High mobility group box protein 1 (HMGB1) was identified as a non-histone DNA-binding protein that functions as a structural compound in the nucleus of somatic cells (1). HMGB1 binds to the minor groove of linear DNA modulating the transcriptional activity of various transcription factors including $\mathrm{p} 53$, nuclear factor $(\mathrm{NF}) \kappa \mathrm{B}$, steroid hormone receptors and homeobox-containing proteins (2). Due to a strong promoter, HMGB1 can be transcribed at extremely

Correspondence to: Dr Babett Bartling, Klinik für Herz- und Thoraxchirurgie, Martin-Luther-Universität Halle-Wittenberg, Ernst-Grube-Str. 40, D-06120 Halle/Saale, Germany

E-mail: babett.bartling@medizin.uni-halle.de

Key words: high mobility group box protein 1, epithelial cells, fibroblasts, lung, cancer high levels, however, a silencer element upstream of the promoter holds HMGB1 transcription at a basal stage under normal conditions (3). Although the expression of HMGB1 is under strict developmental control $(4,5)$ and essential for animal survival (6), HMGB1 is apparent in several mature cells (7-9) as well as in transformed cells $(10,11)$. In cancer cells, HMGB 1 supports cell transformation, growth and cellular resistance (11-13). For this reason, HMGB1 seems to be a crucial factor in cancer development.

The nuclear localization of HMGB1 suggests its main function as a co-transcriptional factor. Moreover, HMGB1 is diffusely distributed in the cytoplasm and can be actively released into the extracellular milieu. Monocytes and macrophages are primarily shown to secrete HMGB 1 in response to inflammatory stimuli (14). However, several other cell types have been identified to store HMGB1 in the cytoplasm $(15,16)$ and subsequently to release HMGB1 depending on the state of the cell (4,17-20). Extracellular HMGB1 acts either as a cytokine-like factor in pathophysiological conditions (reviewed in ref. 21) or as an autocrine/paracrine factor in non-pathophysiological conditions (reviewed in ref. 22). Active secretion of HMGB1 requires the execution of a cellular program independent of the classical secretion pathway via endoplasmic reticulum and Golgi apparatus as HMGB1 lacks the classical secretion signal peptide. Detailed mechanisms responsible for HMGB1 secretion from the cytosol are only partially understood and seem to differ depending on the type of cell $(14,17)$. Besides regulated secretion, HMGB1 can be passively released from the cell upon necrotic cell death thereby triggering systemic inflammation (23). Extracellular HMGB1 molecules mediate their biological function via binding to various interaction partners, such as plasminogen, syndecan-1 and certain cell surface receptors $(24,25)$. One of the HMGB1-binding receptors is RAGE (26), which was first described as a receptor for advanced glycation end products (AGEs) (27). As the multiligand receptor RAGE is highly expressed in lung epithelium, it is suggested as the pivotal surface molecule interacting with extracellular HMGB1 in the lung (26).

The function of epithelial cells in lung tissue can be modified by adjacent fibroblasts of the mesenchyma. Therefore, epithelial-mesenchymal interactions are suggested to play a fundamental role in a number of biological processes including lung development and repair $(28,29)$. As a result of these cell-cell interactions, various regulatory molecules are 
induced that can be classified as transcription factors, signaling molecules, extracellular matrix proteins and their specific receptors (29). In contrast to normal processes, an abnormal activation of fibroblasts destroys the physiological balance between both types of cells and consequently normal tissue function (30-32). In this context, fibroblasts are highly discussed as participating in the development and resistance of epithelial tumors including lung carcinomas (33).

One of the regulatory molecules involved in the very complex process of epithelial-mesenchymal cell interactions may be HMGB1 as it contributes to a large number of processes critical for normal as well as malignant tissues. Therefore, we analyzed the impact of lung fibroblasts on the expression and localization of HMGB1 in lung epithelial cancer cells.

\section{Materials and methods}

Cell lines, culture conditions and treatment. The human lung epithelial cell lines H358, H322 and A549 as well as the primary embryonic lung fibroblasts WI-38 (ATCC cell bank; Manassas, VA, USA) were studied. Moreover, we used H358 cells stably overexpressing RAGE (34). All cells were cultured in Dulbecco's modified Eagle's medium (DMEM) supplemented with $10 \%$ fetal calf serum (FCS), 100 units $/ \mathrm{ml}$ penicillin and $100 \mu \mathrm{g} / \mathrm{ml}$ streptomycin (Invitrogen; Karlsruhe, Germany) at $37^{\circ} \mathrm{C}$ in a $10 \% \mathrm{CO}_{2}$ atmosphere. Three days after culture, conditioned medium was taken from WI-38 fibroblasts and $\mathrm{H} 358$ cells that corresponded to 3.5 to $4 \times 10^{5}$ cells/ml. Moreover, conditioned medium without FCS was generated from confluent WI-38 and H358 cells and finally adjusted with DMEM to $4 \times 10^{5}$ cells/ml. Subconfluent H358 cells were treated for $24 \mathrm{~h}$ with conditioned medium from WI-38 fibroblasts and H358 cells (control), respectively, which was mixed with one volume of fresh DMEM/10\% FCS. For analysis of HMGB1 in the cell culture supernatant, confluent H358 cells were treated with one volume of the FCS-free conditioned medium in fresh DMEM. To detect the passive release of HMGB1 by induction of necrotic cell death, H358 cells were simultaneously incubated with $10 \mathrm{mM}$ hydrogen peroxide and a protease inhibitor mix (Sigma; Deisenhofen, Germany) for $4 \mathrm{~h}$. Mouse monoclonal antibodies against the basic fibroblast growth factor (bFGF; $10 \mu \mathrm{g} / \mathrm{ml}$ clone bFM-1; Upstate; Lake Placid, NY) were applied for neutralizing bFGF in conditioned media.

Protein preparations. Total protein from treated cells was prepared by use of SDS lysis buffer (10 mM Tris-HCl, 2\% SDS, protease inhibitor mix; pH 7.4). Cytoplasmic protein was isolated after permeabilizing the cells in HEPES buffer (20 mM HEPES, $10 \mathrm{mM} \mathrm{NaCl}, 120 \mathrm{mM} \mathrm{KCl,} 1 \mathrm{mM}$ $\mathrm{KH}_{2} \mathrm{PO}_{4}, 1 \mathrm{mM} \mathrm{MgCl} 2,1 \mathrm{mM} \mathrm{Na}{ }_{2}$ EDTA; $\mathrm{pH}$ 7.2) containing $0.5 \%$ nonidet NP-40 and protease inhibitor mix. After $5 \mathrm{~min}$ of incubation on ice, the cytoplasmic protein fraction was cleared by centrifugation. For analysis of extracellular HMGB1, protein was isolated from conditioned medium by use of methanol-chloroform- $\mathrm{H}_{2} \mathrm{O}$ precipitation. Briefly, four volumes methanol, one volume chloroform and three volumes $\mathrm{H}_{2} \mathrm{O}$ were added to the conditioned medium and carefully mixed. After centrifugation at $5000 \times \mathrm{g}$ for $15 \mathrm{~min}$, the interphase plus organic phase was mixed with 3 volumes methanol. The precipitated protein was spun down at $5000 \mathrm{x} \mathrm{g}$ for $15 \mathrm{~min}$ and finally dissolved in SDS lysis buffer. Protein concentration was measured by the BCA protein assay (Pierce; Rockford, IL, USA). For proteolysis of HMGB1, cytoplasmic protein of the cells was isolated in ice-cold incubation buffer $(50 \mathrm{mM}$ Tris- $\mathrm{HCl}, 150 \mathrm{mM} \mathrm{NaCl}, 5 \mathrm{mM}$ $\mathrm{CaCl}_{2}, 0.05 \%$ Brij35; $\left.\mathrm{pH} 7.5\right)$. Cells were disrupted by passages through a 25 G1 needle, and the preparation was cleared by centrifugation. The remaining cytoplasmic fraction was incubated with conditioned medium (as described above) or serum-free solution containing matrix metalloproteinases (MMP)-2 and MMP-9 (AG771; Chemicon; Harrow, UK) for $30 \mathrm{~min}$ at $37^{\circ} \mathrm{C}$ and thereafter stopped by denaturation at $95^{\circ} \mathrm{C}$. Increasing concentrations of $\mathrm{Na}_{2}$ EDTA were added to block MMPs.

Immunoblot analysis. Protein samples were mixed with $4 \mathrm{X}$ loading buffer $(500 \mathrm{mM}$ Tris- $\mathrm{HCl}, 40 \%$ glycerol, $8 \%$ SDS, $80 \mathrm{mM}$ DTT, $4 \mathrm{mM} \mathrm{Na}{ }_{2}$ EDTA, $0.1 \%$ bromophenol blue; $\mathrm{pH}$ 7.4), boiled for $2 \mathrm{~min}$ and immediately subjected to SDS polyacrylamide gel electrophoresis. Thereafter, proteins were electroblotted onto nitrocellulose membranes (Schleicher \& Schüll; Dassel, Germany), blocked with $6 \%$ non-fat dry milk in TBS-T buffer (50 mM Tris- $\mathrm{HCl}, 300 \mathrm{mM} \mathrm{NaCl}, 0.15 \%$ Tween-20; $\mathrm{pH} 7.5$ ) and incubated with the respective primary antibodies. Rabbit polyclonal antibodies against HMGB1 (BD Pharmingen; San Diego, CA, USA) and GAPDH (Abcam; Cambridge, UK) were applied in TBS-T buffer. Primary antibodies were detected by horseradish peroxidaseconjugated secondary antibodies (Dianova; Hamburg, Germany) and subsequently visualized by the enhanced chemiluminescence substrate solution ECL ${ }^{\text {plus }}$ (Amersham; Buckinghamshire, UK). Equal protein loading per lane of the SDS polyacrylamide gel was controlled after staining of the total protein in a coomassie blue staining solution $(50 \%$ methanol, $10 \%$ acetic acid, $0.005 \%$ coomassie blue). All visualized signals were densitometrically estimated by use of the LAS 3000 computer-based imaging system (Fuji Film; Tokyo, Japan) equipped with AIDA 3.5 software (Raytest; Straubenhardt, Germany).

Preparation and expression analysis of RNA. Total RNA was extracted using RNeasy columns (Qiagen, Hilden, Germany). The RNA concentration was calculated from the absorption at $260 \mathrm{~nm}$ by use of the ND-1000 spectrophotometer (NanoDrop; Wilmington, DE, USA) and subsequently reverse transcribed into cDNA with random hexamer primers and Superscript II $^{\mathrm{TM}}$ reverse transcriptase (Invitrogen; Karlsruhe, Germany). Polymerase chain reaction (PCR) was performed with a commercial PCR mix (Promega; Mannheim, Germany) and human $h m g b l$-specific primers (sense: 5' GGA GAG ATG TGG AAT A 3'; antisense: 5' GGG AGT GAG TTG TGT A 3'). An equal quantity of synthesized cDNA was determined by amplification of GAPDH (sense: 5' CAT CAC CAT CTT CCA GGA GCG 3'; antisense: 5' TGA CCT TGC CCA CAG CCT TG $3^{\prime}$ ). PCR was performed after initial denaturation at $95^{\circ} \mathrm{C}$ for $2 \mathrm{~min}, 30 \mathrm{sec}$ at $95^{\circ} \mathrm{C}, 20 \mathrm{sec}$ at primer annealing, and $30 \mathrm{sec}$ at $72^{\circ} \mathrm{C}$ in a Mastercycler ${ }^{\circledR}$ (Eppendorf; Hamburg, Germany). After PCR amplification 

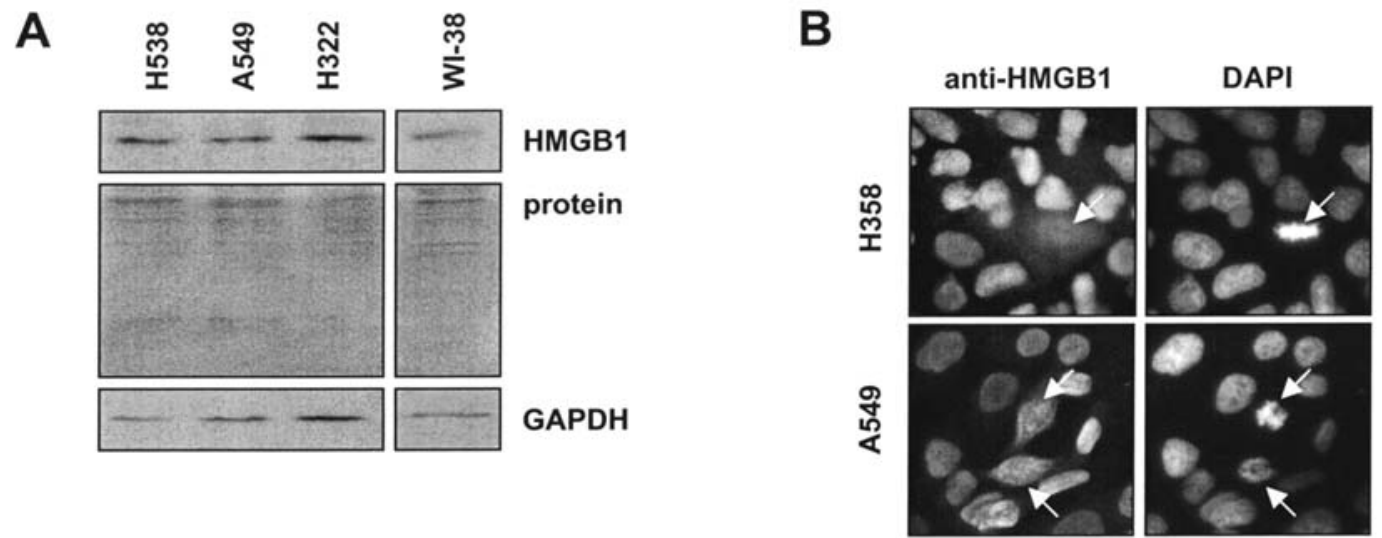

Figure 1. (A) Immunoblot detection of HMGB1 in different human lung epithelial cell lines (H358, A549, H322) and lung fibroblasts (WI-38). Protein loading is shown after coomassie blue staining of the SDS polyacrylamide gel and, additionally, after immunoblot detection of GAPDH. (B) Immunofluorescence staining indicates the cellular localization of HMGB1 in A549 and H358 cells. Condensed nuclear DNA of mitotic cells (arrow) is visible after DAPI staining.
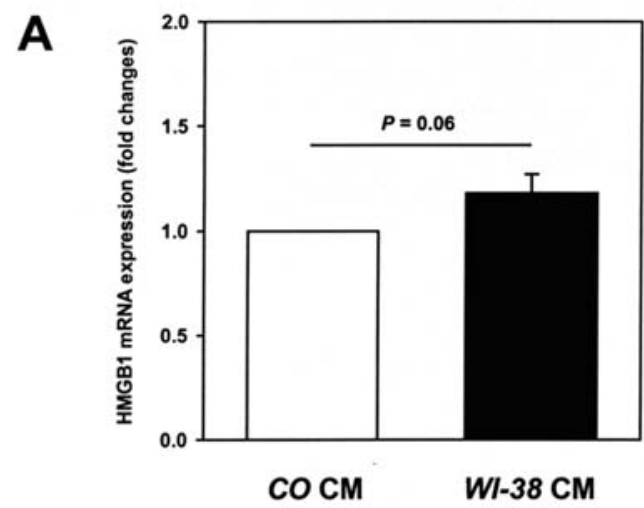

B
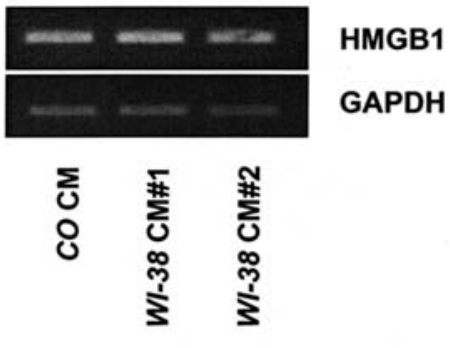

Figure 2. (A) mRNA detection by RT-PCR indicates the level of HMGB1 in H358 cells treated with conditioned medium of fibroblasts (WI-38 CM) compared with conditioned medium from control H358 cells (CO CM). All data are given as the mean \pm SEM ( $\mathrm{n}=5$ ). (B) Representative PCR amplifications of HMGB 1 are shown for two different preparations of conditioned medium from fibroblasts (WI-38 CM\#1, \#2) and control conditioned medium (CO CM). RT-PCR of GAPDH mRNA indicates equal RNA loading per lane. CM, conditioned medium.

and gel electrophoretic separation, the intensity of all PCR products was evaluated using the LAS 3000 computer-based imaging system and AIDA 3.5 software. Prior to quantitative analyses, the cycle-to-cycle efficiency of the PCR amplification was assessed ensuring PCR investigations in the exponential phase of the reaction. Finally, the mRNA expression values were calculated as the ratio per $18 \mathrm{~S}$ rRNA.

Immunocytochemistry. Cells grown on glass coverslips were washed with PBS, fixed in phosphate-buffered $4 \%$ formaldehyde for $10 \mathrm{~min}$ at $4{ }^{\circ} \mathrm{C}$. After membrane permeabilization with $0.5 \%$ Nonidet NP-40, cells were blocked with $10 \%$ goat serum albumin and stained for $1 \mathrm{~h}$ with the primary rabbit HMGB1 antibody (1:100 in PBS) at room temperature. Antibody detection was performed with the goat Alexa Fluor anti-rabbit IgG secondary antibody (Molecular Probes Europe; Leiden, Netherlands) and fluorescence microscopy equipped with a Spot RT camera (Zeiss; Jena, Germany). Moreover, RAGE was detected by immunocytochemistry in accordance with the protocol described previously (34). Omission of the primary antibody served as a negative control. Nuclei were visualized by DNA staining with the fluorochrome 4',6-diamidino-2-phenylindole dihydrochloride (DAPI; Molecular Probes Europe). Slides were embedded in Mowiol mounting medium (Merck; Bad Soden, Germany).

Statistics. The significance of comparison of the mean values was determined by the Student's t-test (SigmaStat software; Jandel Corp., San Rafael, CA, USA). Data reported are the means \pm SEM with $\mathrm{P} \leq 0.05$ indicating a significant difference of mean data.

\section{Results}

HMGB1 expression and localization in human lung epithelial cells and fibroblasts. Protein analyses of several lung epithelial cell lines revealed HMGB1 expression in H358, H322 and A549 cells (Fig. 1A). Subsequent immunocytochemistry indicated the primary location of HMGB1 in the nucleus of these epithelial cells. In contrast, the cytoplasmic localization of HMGB1 was less detectable in quiescent cells but prominent in mitotic cells (Fig. 1B). The primary lung 
A

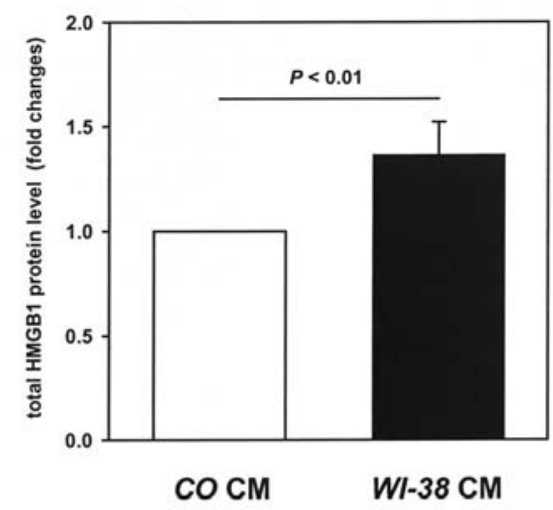

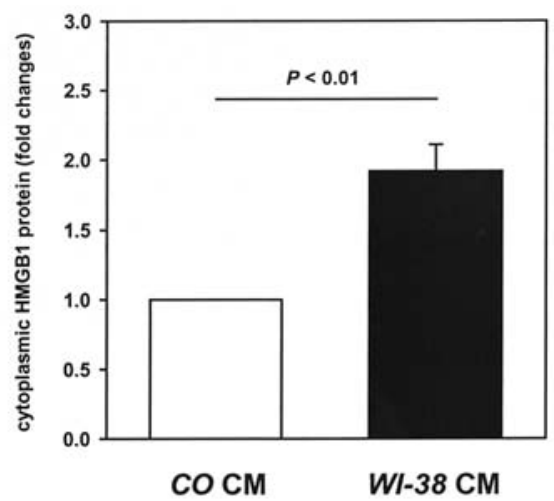

$\operatorname{cocm}$
WI-38 CM

B

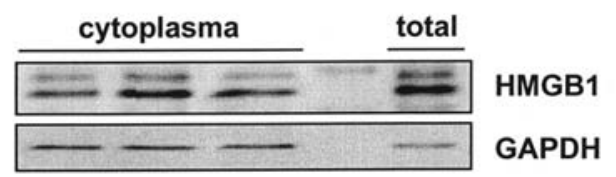

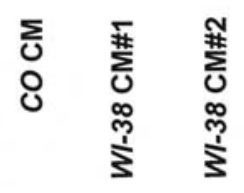

Figure 3. (A) Impact of conditioned medium from fibroblasts (WI-38 CM) on the level of total and cytoplasmic HMGB1 protein in H358 cells compared with conditioned medium from control H358 cells $(\mathrm{CO} \mathrm{CM})$. All data are the means \pm SEM $(\mathrm{n}=4)$. (B) Representative HMGB1 immunoblots of the cytoplasmic protein fraction are shown for two different preparations of conditioned medium from fibroblasts (WI-38 CM\#1, \#2) and control conditioned medium (CO $\mathrm{CM}$ ). Total protein was loaded as a positive control. GAPDH detection indicates the protein loading per lane. CM, conditioned medium.

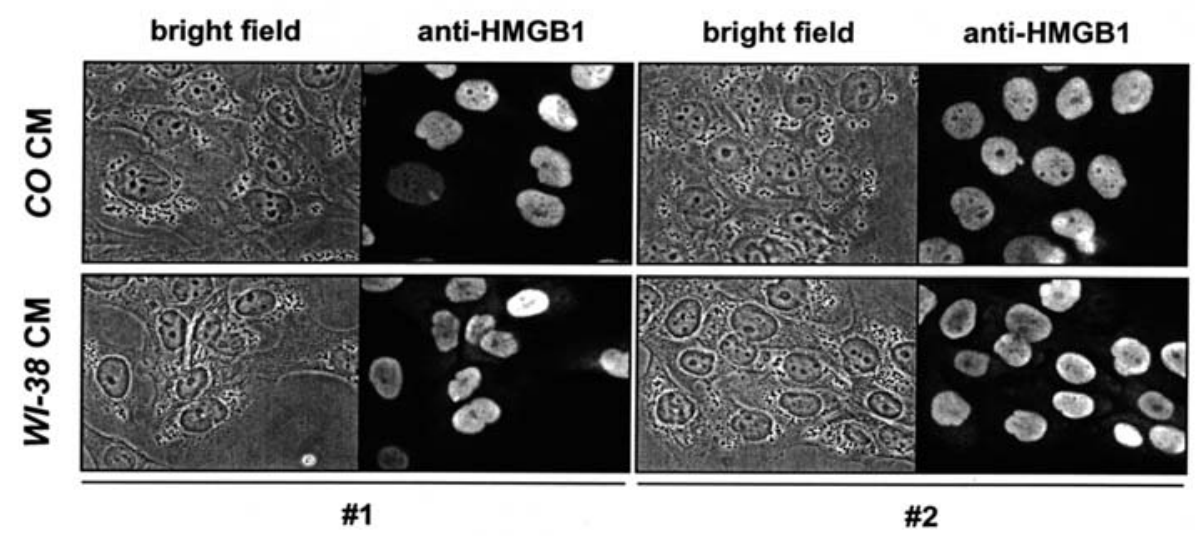

Figure 4. Immunofluorescence staining of HMGB1 in the nucleus and partially in the cytoplasm after treatment of H358 cells with two preparations (\#1, \#2) of control conditioned medium $(C O \mathrm{CM})$ and conditioned medium from WI-38 fibroblasts (WI-38 CM). The respective bright field images are shown to the left. CM, conditioned medium.

fibroblasts WI-38 also expressed HMGB1 protein (Fig. 1A), which was mainly localized in the nucleus as well (data not shown).

Impact of fibroblasts on the level of HMGBI in lung epithelial cells. We used the cell line $\mathrm{H} 358$ for further studies on the influence of fibroblasts on epithelial HMGB1. As summarized in Fig. 2, cultivation of H358 cells in conditioned medium from WI-38 fibroblasts resulted in a slightly increased mRNA expression of HMGB1. This moderate up-regulation of HMGB1 mRNA was confirmed at the protein level by analyzing total protein fractions (Fig. 3A). However, the individual isolation of cytoplasmic protein fractions revealed a stronger increase in HMGB1 located in the cytoplasm of H358 cells (Fig. 3). Subsequent immunocytochemistry also indicated a higher amount of cytoplasmic HMGB1 in response to conditioned medium from WI-38 fibroblasts compared to control conditioned medium (Fig. 4). This observation was independent of the nucleo-cytoplasmic distribution of HMGB1 during mitosis (compare to Fig. 1B). The additional incubation of $\mathrm{H} 358$ cells with an antibody that blocks the basic fibroblast growth factor (bFGF) impaired the 

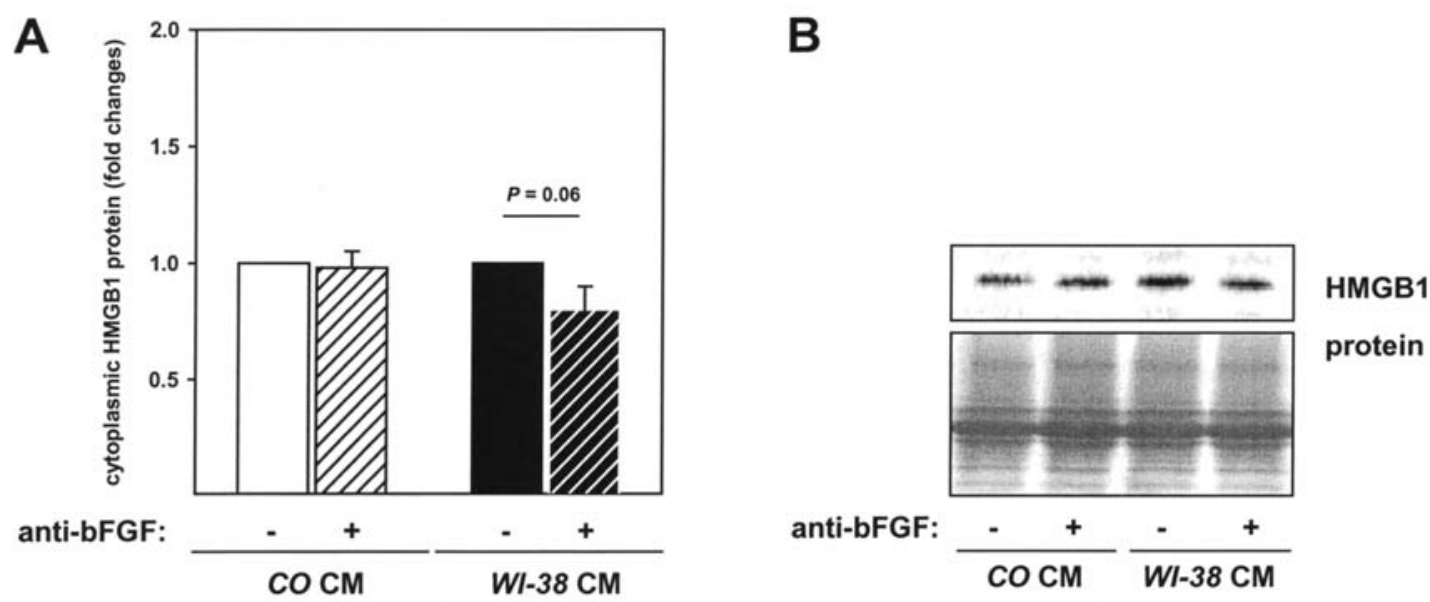

Figure 5. (A) Level of cytoplasmic HMGB1 protein in $\mathrm{H} 358$ cells in response to conditioned medium from fibroblast (WI-38 $\mathrm{CM}$ ) or control $\mathrm{H} 358$ cells $(C O$ $\mathrm{CM}$ ), which was simultaneously treated with a blocking anti-bFGF antibody. All data are the means \pm SEM (n=6). (B) HMGB1 immunoblots of the cytoplasmic protein fraction are shown for representative samples incubated with and without the anti-bFGF-treated conditioned medium. Protein staining of the SDS polyacrylamide gel indicates equal protein loading per lane. CM, conditioned medium.

level of cytoplasmic HMGB1, which was only observed for the conditioned medium from WI-38 fibroblasts but not the conditioned medium from H358 controls (Fig. 5).

Extracellular HMGB1 of lung epithelial cells. As cytoplasmic HMGB1 can be secreted, we also studied HMGB1 in cell culture supernatants of $\mathrm{H} 358$ cells. Firstly, we analyzed the basic level of HMGB1 in conditioned media from WI-38 and H358 cells but did not find a detectable amount of free extracellular HMGB1 (data not shown). One reason could have been the fast degradation of HMGB 1 by extracellular proteases. To test this hypothesis, we investigated the impact of conditioned media and matrix metalloproteinases (MMPs) on HMGB 1 degradation. Indeed, cytoplasmic HMGB 1 protein was highly degraded by preparations containing MMP-2/-9 as well as by conditioned media containing serum, which was delayed by adding EDTA (Fig. 6A).

As the culturing of $\mathrm{H} 358$ cells in the presence of EDTA or general protease inhibitors greatly affected the viability and HMGB1 release (data not shown), we established serum-free conditioned media from WI-38 fibroblasts and H358 cells which had been preincubated at $37^{\circ} \mathrm{C}$ for $1 \mathrm{~h}$ to allow the complete degradation of potential extracellular HMGB1 ( $0 \mathrm{~h}$; Fig. 6B). The subsequent treatment of H358 cells with these medium preparations resulted in a very small amount of free extracellular HMGB1 in response to conditioned medium from fibroblasts compared with control medium (24 h; Fig. 6B). However, despite the excessive amount of treated $\mathrm{H} 358$ cells, we identified in a minor number of experiments a detectable level of extracellular HMGB1. In contrast, necrotic cell death induction with hydrogen peroxide induced an apparent release of HMGB1 $\left(\mathrm{H}_{2} \mathrm{O}_{2}\right.$; Fig. 6B). As these data suggested a negligible amount of secreted HMGB1 in physiological conditions, we additionally investigated H358 cells overexpressing the HMGB1-binding surface receptor RAGE. Costaining of non-nuclear HMGB1 and RAGE by immunofluorescence identified only few co-localization events of HMGB1 and RAGE in single cells but no co-localization events in confluent cells (Fig. 6C).

\section{Discussion}

Stromal fibroblasts of the lung tissue can variably affect the function of lung epithelial cells (29). Depending on their cellular state, fibroblasts are suggested to stabilize the function of the epithelium in normal conditions but alter its function in pathophysiological conditions. In lung cancer and other carcinomas, fibroblasts have been shown to contribute to the initiation, progression and resistance of tumors $(33,35)$. Our previous research supported these results as lung fibroblasts caused a mitogenic stimulation of the lung epithelial cell line H358 (34). This was not only associated with an enhanced proliferation but also with less spontaneous cell death (36).

By using the same experimental model, we now revealed that fibroblast factors cause an increase in HMGB1 at the mRNA and protein level in H358 lung carcinoma cells. As HMGB1 is a direct c-Myc target gene involved in the Mycmediated neoplastic transformation and cellular proliferation $(12,37,38)$, our data emphasizes the potential relation between epithelial-mesenchymal interactions and HMGB1 in cancer development. Although the expression of HMGB1 is not up-regulated in human lung carcinomas (39) contrasting with other types of cancer $(11,13)$, HMGB1 was already detectable at a basal level in each studied lung cancer cell line.

On the basis of this and the further increase in HMGB1 in response to fibroblasts, our data also support the potential role of HMGB1 in mediating cellular resistance. In this context, it has been shown that not only fibroblast growth factors (40-43) but also HMGB1 protects somatic cells against different death stimuli including ultraviolet radiation, chemotherapeutics and caspase-induced apoptosis $(10,11,13)$. One critical downstream mechanism involved in this HMGB1-mediated cell resistance is the activation of NFKB and subsequent stimulation of the $\mathrm{NF \kappa B}$ target gene product c-IAP2, a potent inhibitor of the caspases -9 and -3 (13).

As mentioned above, fibroblasts already up-regulated HMGB1 at the mRNA level indicating that gene transcription and/or mRNA stability of HMGB1 contribute to its increased 

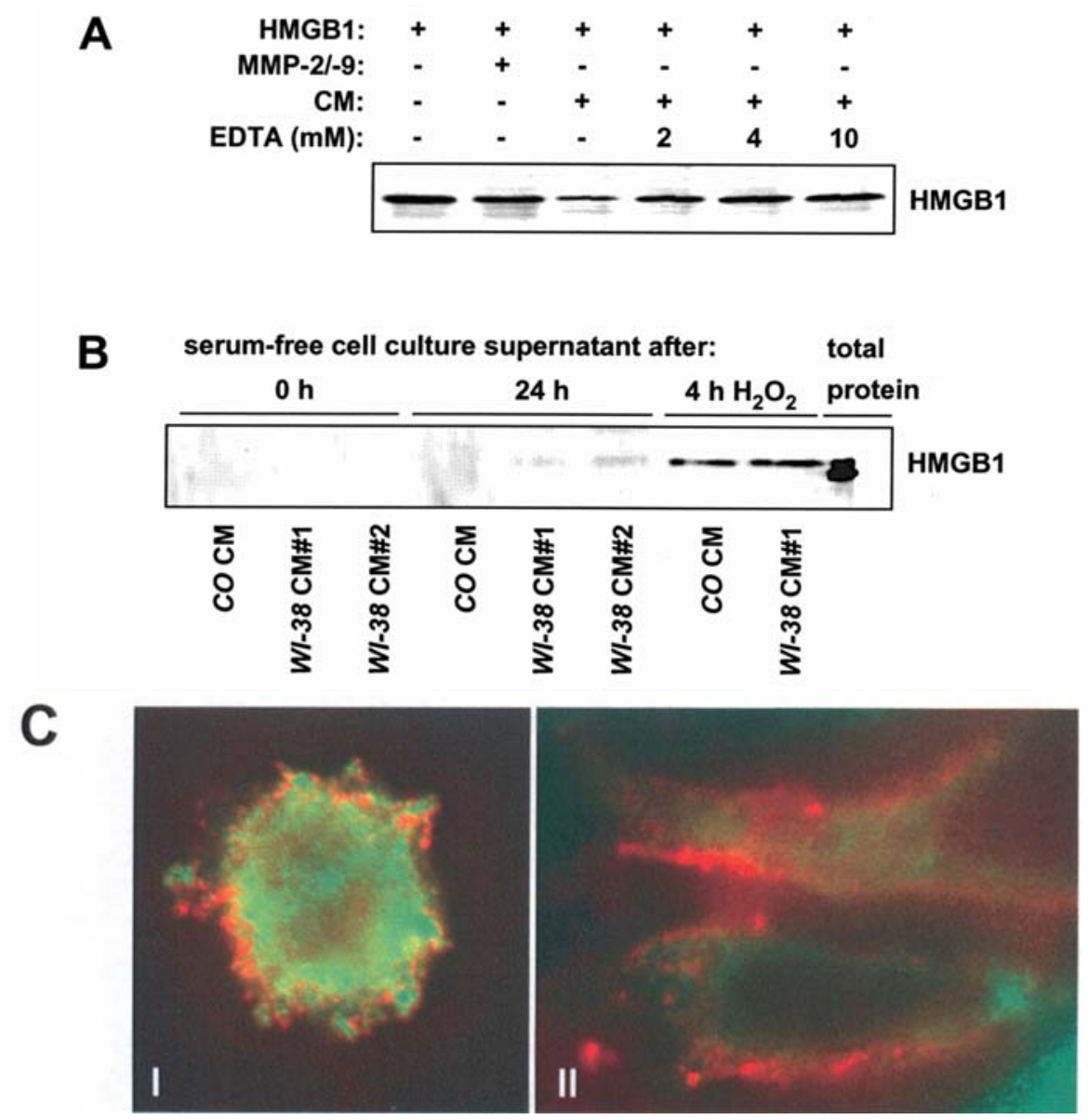

Figure 6. (A) Immunoblot of the cytoplasmic fraction containing HMGB1 protein which was incubated without and with MMP-2/-9 solution or cell culture supernatant (i.e. conditioned medium; CM) in the presence of EDTA for $30 \mathrm{~min}$ at $37^{\circ} \mathrm{C}$. (B) Impact of two different preparations of serum-free conditioned medium from fibroblasts (WI-38 CM \#1, \#2) compared with control conditioned medium (CO CM) on the extracellular level of HMGB1 after 24 h of treatment and after additional treatment with hydrogen peroxide for $4 \mathrm{~h}$ to generate positive controls $\left(\mathrm{H}_{2} \mathrm{O}_{2}\right.$; in the presence of protease inhibitor mix). The protein precipitated from the respective cell culture supernatant corresponds to $\sim 10^{6} \mathrm{H} 358$ cells per lane. The analogous protein amount of the conditioned medium applied for the experiment (CO CM and WI-38 CM\#1, \#2) was additionally loaded as negative control showing no detectable signal of extracellular HMGB1 at the beginning of the treatment $(0 \mathrm{~h})$. H358 total protein was loaded as another positive control. (C) Immunofluorescence staining of non-nuclear HMGB1 (green), RAGE (red) and HMGB1-RAGE (yellow; overlay of the images) in RAGE overexpressing H358 cells after $24 \mathrm{~h}$ of treatment with conditioned medium from WI-38 fibroblasts. H358 cells were formaldehyde-fixed without additional cell permeabilization by detergents to prevent the excessive staining of nuclear HMGB1 (images I and II).

protein level in H358 lung epithelial cells. Although several transcription factors (p73a, nuclear factor-1, CCAAT-binding transcription factor-2 and STAT transcription factors) as well as the abundance of three mRNA transcripts have been reported (44), it is not yet clear which are likely to regulate the level of epithelial HMGB1 due to diffusible factors of fibroblasts.

The primary detection of HMGB 1 in the nucleus of several lung epithelial cell lines emphasizes the major function of HMGB1 as a chromatin compound. As HMGB1 modulates a large number of DNA-binding factors (2), an increased level of HMGB1 might cause multiple cellular changes. Moreover, we demonstrated that HMGB1 is localized in the cytoplasm of the lung epithelial cells, particularly during mitosis of the cells. Our observations are consistent with recent publications demonstrating that HMGB1 is not only present as a DNA-bound but also as a free form, which dissociates from chromosomes during mitosis (45). Correspondingly, nuclear HMGB1 is absent during DNA replication in the regenerating liver and primary hepatoma (16).
The nucleo-cytoplasmic distribution of HMGB1 is tightly controlled by two nuclear localization signals, two putative nuclear export signals $(46,47)$ and posttranslational phosphorylations (48). HMGB1 can be phosphorylated by distinct protein kinases (PK) $\left[\mathrm{Ca}^{2+}\right.$-phospholipid-dependent PK (49), PKCs $\alpha / \beta / \gamma$ and creatine kinase I (50)], and the phosphorylation of HMGB 1 directs its cellular distribution towards the cytoplasm (48). In addition, in lung epithelial cells, fibroblast factors caused a stronger increase in HMGB1 in the cytoplasmic compared to the total protein level. Although fibroblast factors provoke numerous phosphorylation events in epithelial cells, such as the phosphorylation of the protein kinases Akt and p70S6 (36), current literature has not yet revealed a potential role of HMGB1-specific kinases in similar experimental models. Besides it is largely unknown whether HMGB 1 executes active processes in the cytoplasm.

The relative increase of the cytoplasmic per total HMGB1 protein may, at least in part, result from the dissociation of nuclear HMGB 1 into the cytoplasm during mitosis of the epithelial cells $(16,51)$. Our previous study clearly demonstrated a greater $(\sim 1.2$-fold $)$ proliferation of lung 
epithelial cells in the presence of fibroblasts and conditioned medium from fibroblasts, respectively, which was less pronounced by inhibiting the basic fibroblast growth factor (bFGF) (34). Due to this, the enhanced level of cytoplasmic HMGB1 was diminished by simultaneous blockade of bFGF. Nonetheless, the increased amount of the cytoplasmic HMGB1 was higher than the increased number of proliferating cells in conditioned media from the fibroblasts, suggesting further mechanisms, in addition to mitosis, that influence the nucleo-cytoplasmic distribution of HMGB1.

The cellular localization of HMGB1 is not only modulated by posttranslational phosphorylation but also by acetylation. The acetylation of cytoplasmic HMGB1 provokes its storage in secretory lysosomes with subsequent HMGB1 release via exocytosis (47). Although this kind of secretion is rather specific for activated immune cells, HMGB1 can also be exported from other types of mammalian cells by still unknown mechanisms (4,17-20). For that reason, we examined HMGB1 in the culture medium of the H358 lung epithelial cells but found no significant amount of soluble HMGB1 in our experimental model. In some experiments we determined an extremely small amount of extracellular HMGB1, which results from cells upon necrotic cell death in vitro. As HMGB1 might be locally exported to the surface of cells without the extensive appearance of soluble HMGB1, we additionally studied $\mathrm{H} 358$ cells overexpressing the HMGB1binding receptor RAGE (27). Although extracellular HMGB1 interacts with a variety of surface molecules (22), the overexpression of RAGE might direct HMGB1 binding towards this receptor. However, immunocytochemisty determined rare co-localization events of RAGE and HMGB1 suggesting the primary localization of non-nuclear HMGB1 in the cytoplasm of the $\mathrm{H} 358$ cells in these experimental conditions. In spite of our observation, it is possible that HMGB1 can be exported from lung epithelial cells in response to stimuli other than the fibroblast factors.

Another reason for the low incidence of extracellular HMGB1 in the lung epithelial cells might be based on the fact that HMGB1 undergoes rapid proteolysis in cell culture medium. Similar results have been published for the culture medium of murine erythroleukemia cells (20) and in response to the tissue-type plasminogen activator/plasminogen system in neuroblastoma cells (25). As epithelial lung cancer cells and fibroblasts secrete matrix metalloproteinases (MMPs), such as MMP-2 (52), and the HMGB1 proteolysis was delayed by adding MMP-inhibitory EDTA, soluble HMGB1 may be an MMP target protein. This assumption was confirmed by another experiment demonstrating the strong degradation of cytoplasmic HMGB1 in the presence of MMP-2/-9. The active $10-\mathrm{kDa}$ HMGB 1 fragment generated by a serine protease (20) was not detected in the culture medium of H358 lung epithelial cells.

In summary, diffusible factors of fibroblasts enhance the expression of HMGB1 in lung epithelial cancer cells suggesting an important role of HMGB1 in the fibroblastmediated growth and resistance of lung carcinomas. As epithelial-mesenchymal interactions do not only contribute to tumor biology, the increased level of the multi-functional protein HMGB1 may modify further biological processes of lung tissue supposedly driven by stromal fibroblasts.

\section{Acknowledgements}

This project was supported by Wilhelm Roux grants of the Bundesministerium für Bildung und Forschung, BMBF (FKZ13/0 and FKZSI/07), by the Deutsche Krebshilfe (107078) and the Deutsche Forschungsgemeinschaft, DFG (Si-1317/1-1).

\section{References}

1. Goodwin GH, Sanders C and Johns EW: A new group of chromatin-associated proteins with a high content of acidic and basic amino acids. Eur J Biochem 38: 14-19, 1973.

2. Bustin M: Regulation of DNA-dependent activities by the functional motifs of the high-mobility-group chromosomal proteins. Mol Cell Biol 19: 5237-5246, 1999.

3. Lum HK and Lee KL: The human HMGB 1 promoter is modulated by a silencer and an enhancer-containing intron. Biochim Biophys Acta 1520: 79-84, 2001.

4. Rauvala $\mathrm{H}$ and Pihlaskari R: Isolation and some characteristics of an adhesive factor of brain that enhances neurite outgrowth in central neurons. J Biol Chem 262: 16625-16635, 1987.

5. Zhao Z, Chou DK, Nair SM, Tobet S and Jungalwala FB: Expression of sulfoglucuronyl (HNK-1) carbohydrate and its binding protein (SBP-1) in developing rat cerebellum. Brain Res Dev Brain Res 120: 165-180, 2000.

6. Calogero S, Grassi F, Aguzzi A, et al: The lack of chromosomal protein Hmg 1 does not disrupt cell growth but causes lethal hypoglycaemia in newborn mice. Nat Genet 22: 276-280, 1999.

7. Degryse B, Bonaldi T, Scaffidi P, et al: The high mobility group (HMG) boxes of the nuclear protein HMG1 induce chemotaxis and cytoskeleton reorganization in rat smooth muscle cells. J Cell Biol 152: 1197-1206, 2001.

8. Fiuza C, Bustin M, Talwar S, et al: Inflammation-promoting activity of HMGB1 on human microvascular endothelial cells. Blood 101: 2652-2660, 2003.

9. Wang H, Yang H and Tracey KJ: Extracellular role of HMGB1 in inflammation and sepsis. J Intern Med 255: 320-331, 2004.

10. Nagatani G, Nomoto M, Takano H, et al: Transcriptional activation of the human HMG1 gene in cisplatin-resistant human cancer cells. Cancer Res 61: 1592-1597, 2001.

11. Brezniceanu ML, Volp K, Bosser S, et al: HMGB1 inhibits cell death in yeast and mammalian cells and is abundantly expressed in human breast carcinoma. FASEB J 17: 1295-1297, 2003.

12. Wood LJ, Maher JF, Bunton TE and Resar LM: The oncogenic properties of the HMG-I gene family. Cancer Res 60: 4256-4261, 2000.

13. Volp K, Brezniceanu ML, Bosser S, et al: Increased expression of high mobility group box 1 (HMGB1) is associated with an elevated level of the antiapoptotic c-IAP2 protein in human colon carcinomas. Gut 55: 234-242, 2006.

14. Gardella S, Andrei C, Ferrera D, et al: The nuclear protein HMGB1 is secreted by monocytes via a non-classical, vesiclemediated secretory pathway. EMBO Rep 3: 995-1001, 2002.

15. Bustin $\mathrm{M}$ and Neihart NK: Antibodies against chromosomal HMG proteins stain the cytoplasm of mammalian cells. Cell 16: 181-189, 1979.

16. Mosevitsky MI, Novitskaya VA, Iogannsen MG and Zabezhinsky MA: Tissue specificity of nucleo-cytoplasmic distribution of HMG1 and HMG2 proteins and their probable functions. Eur J Biochem 185: 303-310, 1989.

17. Fages C, Nolo R, Huttunen HJ, Eskelinen E and Rauvala H: Regulation of cell migration by amphoterin. J Cell Sci 113: 611-620, 2000.

18. Rouhiainen A, Imai S, Rauvala H and Parkkinen J: Occurrence of amphoterin (HMG1) as an endogenous protein of human platelets that is exported to the cell surface upon platelet activation. Thromb Haemost 84: 1087-1094, 2000.

19. Charoonpatrapong K, Shah R, Robling AG, et al: HMGB1 expression and release by bone cells. J Cell Physiol 207: 480-490, 2006.

20. Sparatore B, Patrone M, Passalacqua M, et al: Extracellular processing of amphoterin generates a peptide active on erythroleukaemia cell differentiation. Biochem J 357: 569-574, 2001.

21. Ulloa L and Messmer D: High-mobility group box 1 (HMGB1) protein: friend and foe. Cytokine Growth Factor Rev 17: 189-201, 2006. 
22. Huttunen HJ and Rauvala H: Amphoterin as an extracellular regulator of cell motility: from discovery to disease. J Intern Med 255: 351-366, 2004.

23. Scaffidi P, Misteli T and Bianchi M: Release of chromatin protein HMGB1 by necrotic cells triggers inflammation. Nature 418: 191-195, 2002.

24. Salmivirta M, Rauvala H, Elenius K and Jalkanen M: Neurite growth-promoting protein (amphoterin, p30) binds syndecan. Exp Cell Res 200: 444-451, 1992.

25. Parkkinen $J$ and Rauvala H: Interactions of plasminogen and tissue plasminogen activator (t-PA) with amphoterin. Enhancement of t-PA-catalyzed plasminogen activation by amphoterin. J Biol Chem 266: 16730-16735, 1991.

26. Hori O, Brett J, Slattery T, et al: The receptor for advanced glycation end products (RAGE) is a cellular binding site for amphoterin. Mediation of neurite outgrowth and co-expression of rage and amphoterin in the developing nervous system. J Biol Chem 270: 25752-25761, 1995.

27. Neeper M, Schmidt A, Brett J, et al: Cloning and expression of a cell surface receptor for advanced glycosylation end products of proteins. J Biol Chem 267: 14998-15004, 1992.

28. Alescio $\mathrm{T}$ and Cassini A: Induction in vitro of tracheal buds by pulmonary mesenchyme grafted on tracheal epithelium. J Exp Zool 150: 83-94, 1962.

29. Demayo F, Minoo P, Plopper CG, Schuger L, Shannon J and Torday JS: Mesenchymal-epithelial interactions in lung development and repair: are modeling and remodeling the same process? Am J Physiol Lung Cell Mol Physiol 283: L510-L517, 2002.

30. Garantziotis S, Steele MP and Schwartz DA: Pulmonary fibrosis: thinking outside of the lung. J Clin Invest 114: 319-321, 2004.

31. Selman $\mathrm{M}$ and Pardo A: Idiopathic pulmonary fibrosis: an epithelial/fibroblastic cross-talk disorder. Respir Res 3: 3, 2002.

32. Knight D: Epithelium-fibroblast interactions in response to airway inflammation. Immunol Cell Biol 79: 160-164, 2001.

33. Wernert N: The multiple roles of tumour stroma. Virchows Arch 430: 433-443, 1997.

34. Bartling B, Demling N, Silber RE and Simm A: Proliferative stimulus of lung fibroblasts on lung cancer cells is impaired by RAGE. Am J Respir Cell Mol Biol 34: 83-91, 2006.

35. Micke P and Ostman A: Tumour-stroma interaction: cancerassociated fibroblasts as novel targets in anti-cancer therapy? Lung Cancer 45 (suppl 2): 163-175, 2004.

36. Bartling B, Rehbein G, Silber RE and Simm A: Senescent fibroblasts induce moderate stress in lung epithelial cells in vitro. Exp Gerontol 41: 532-539, 2006.

37. Wood LJ, Mukherjee M, Dolde CE, et al: HMG-I/Y, a new c-Myc target gene and potential oncogene. Mol Cell Biol 20: 5490-5502, 2000.

38. Yoon S, Lee JY, Yoon BK, Bae DS and Choi DS: Effects of HMGB-1 overexpression on cell-cycle progression in MCF-7 cells. J Korean Med Sci 19: 321-326, 2004.
39. The cancer genome anatomic project. cgap.nci.nih.gov/SAGE/ AnatomicViewer.

40. Cohen-Jonathan E, Toulas C, Monteil S, et al: Radioresistance induced by the high molecular forms of the basic fibroblast growth factor is associated with an increased G2 delay and a hyperphosphorylation of p34CDC2 in HeLa cells. Cancer Res 57: 1364-1370, 1997.

41. Konig A, Menzel T, Lynen S, et al: Basic fibroblast growth factor (bFGF) upregulates the expression of bcl-2 in B cell chronic lymphocytic leukemia cell lines resulting in delaying apoptosis. Leukemia 11: 258-265, 1997.

42. Miyake H, Hara I, Gohji K, Yoshimura K, Arakawa S and Kamidono S: Expression of basic fibroblast growth factor is associated with resistance to cisplatin in a human bladder cancer cell line. Cancer Lett 123: 121-126, 1998.

43. Muerkoster S, Wegehenkel K, Arlt A, et al: Tumor stroma interactions induce chemoresistance in pancreatic ductal carcinoma cells involving increased secretion and paracrine effects of nitric oxide and interleukin-1beta. Cancer Res 64: 1331-1337, 2004.

44. Muller S, Ronfani L and Bianchi ME: Regulated expression and subcellular localization of HMGB1, a chromatin protein with a cytokine function. J Intern Med 255: 332-343, 2004.

45. Falciola L, Spada F, Calogero S, et al: High mobility group 1 protein is not stably associated with the chromosomes of somatic cells. J Cell Biol 137: 19-26, 1997.

46. Wen L, Huang JK, Johnson BH and Reeck GR: A human placental cDNA clone that encodes nonhistone chromosomal protein HMG-1. Nucleic Acids Res 17: 1197-1214, 1989.

47. Bonaldi T, Talamo F, Scaffidi P, et al: Monocytic cells hyperacetylate chromatin protein HMGB 1 to redirect it towards secretion. EMBO J 22: 5551-5560, 2003.

48. Youn JH and Shin JS: Nucleocytoplasmic shuttling of HMGB1 is regulated by phosphorylation that redirects it toward secretion. J Immunol 177: 7889-7897, 2006.

49. Ramachandran C, Yau P, Bradbury EM, Shyamala G, Yasuda H and Walsh DA: Phosphorylation of high-mobility-group proteins by the calcium-phospholipid-dependent protein kinase and the cyclic AMP-dependent protein kinase. J Biol Chem 259: $13495-13503,1984$.

50. Sakamoto R, Okano M, Takena $\mathrm{H}$ and Ohtsuki K: Inhibitory effect of glycyrrhizin on the phosphorylation and DNA-binding abilities of high mobility group proteins 1 and 2 in vitro. Biol Pharm Bull 24: 906-911, 2001.

51. Pallier C, Scaffidi P, Chopineau-Proust S, et al: Association of chromatin protein high mobility group box (HMGB) 1 and HMGB2 with mitotic chromosomes. Mol Biol Cell 14: 3414-3426, 2003.

52. Yoshizaki T, Sato H and Furukawa M: Recent advances in the regulation of matrix metalloproteinase 2 activation: From basic research to clinical implication. Oncol Rep 9: 607-611, 2002. 